\title{
Fontan at 40: Midlife crisis?
}

\author{
Carl L. Backer, MD, ${ }^{a}$ and Marshall L. Jacobs, $\mathrm{MD}^{\mathrm{b}}$
}

\author{
From the ${ }^{a}$ Division of Cardiovascular-Thoracic Surgery, Ann \& Robert H. Lurie Children's Hospital of Chicago, \\ Chicago, Ill; and ${ }^{\mathrm{b}}$ Department of Cardiac Surgery, Johns Hopkins University, Baltimore, Md. \\ Disclosers: Authors have nothing to disclose with regard to commercial support. \\ Received for publication Sept 4, 2015; accepted for publication Sept 8, 2015. \\ Address for reprints: Carl L. Backer, MD, Division of Cardiovascular-Thoracic Surgery, Ann \& Robert H. Lurie \\ Children's Hospital of Chicago, 225 E Chicago Ave, Chicago, IL 60611 (E-mail: cbacker@luriechildrens.org). \\ J Thorac Cardiovasc Surg 2016;151:621-2 \\ $0022-5223 / \$ 36.00$ \\ Copyright (C) 2016 by The American Association for Thoracic Surgery \\ http://dx.doi.org/10.1016/j.jtcvs.2015.09.117
}

The cardiac surgical procedure that today is widely known as the Fontan operation was introduced separately by Francis Fontan and Guillermo Kreutzer in the early 1970s and originally was only applied to patients with tricuspid atresia. ${ }^{1,2}$ Over the past 4 decades, this surgical procedure has evolved considerably. It is now used more and more often for patients with a spectrum of complex congenital heart diseases sharing the common feature of a functionally univentricular heart, including hypoplastic left heart syndrome and various anomalies associated with heterotaxy syndromes. At Ann \& Robert H. Lurie Children's Hospital of Chicago, palliative operations leading up to the Fontan, the Fontan operation itself, and reoperations for patients who have undergone the Fontan operation account for $17 \%$ of our total pediatric cardiac cases. In many respects the Fontan operation, along with preparatory and post-Fontan procedures, have become signature operations for pediatric cardiac surgeons. The resources devoted by heart center teams to the pre- and postoperative care of these patients in their varying stages are quite enormous.

Careful analysis of outcomes and clinically modifiable variables in patients who have undergone a Fontan procedure and attempts to improve the outcomes of these patients have been the focus of innumerable publications. Despite these efforts, patients undergoing Fontan procedures continue to develop late sequelae of arrhythmias, ventricular dysfunction, atrioventricular valve insufficiency, cirrhosis, protein-losing enteropathy, plastic bronchitis, ascites, and exercise intolerance. Within Lurie Children's Heart Transplant Program, the fraction of all heart transplant recipients who have failure of univentricular circulation-either en route to a Fontan operation or post-Fontan-has increased from $12 \%$ in the early 1990 s to $36 \%$ in the past 5 years.

Preventing these late sequelae is extraordinarily important. It seems intuitively obvious that improvements in preparing patients for the Fontan procedure and refinements in technique would combine to decrease these late sequelae. One might also speculate that there would be convergence to the mean on surgical strategies and techniques. However, despite 40 years of experience, we

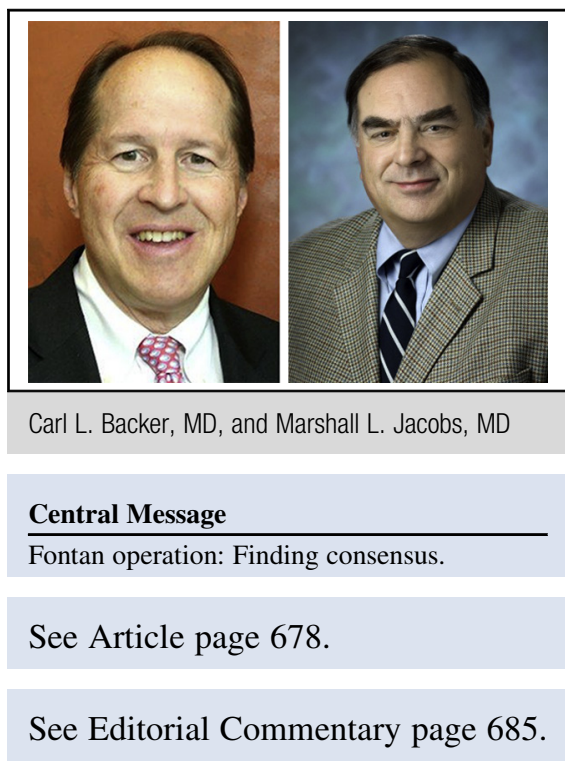

are nowhere near consensus on how to optimally prepare patients for a Fontan operation or even which Fontan operation is best.

Because of the normally elevated pulmonary vascular resistance in infants, a Fontan operation is not generally performed until after age 18 months. Hence, nearly all of these patients will require some form of initial surgical palliation, the nature of which is still unresolved. Options, which depend on the specific morphology and physiology of the patient, include modified Blalock-Taussig shunt (different shunt sizes), pulmonary artery band, and ductal stenting. For patients with a single ventricle with systemic ventricular outflow obstruction and aortic arch obstruction, the Norwood operation has become a standard procedure. However, within this subgroup of patients there is still a question of whether we should use the Sano modification (right ventricle to pulmonary artery shunt) or modified Blalock-Taussig shunt. Alternatively, some centers favor a hybrid strategy with initial bilateral pulmonary artery banding and ductal stenting. There is no consensus on initial palliation.

It appears that bidirectional superior cavopulmonary anastomosis has become a standard staging procedure before a Fontan operation. This procedure unloads the volume presented to the single ventricle while providing acceptable systemic oxygen saturations. However, there are 2 different ways of doing a bidirectional superior cavopulmonary anastomosis: hemi-Fontan or direct cavopulmonary anastomosis. Also in question is the timing of the bidirectional superior cavopulmonary anastomosis: 
3 months, 4 months, or 6 months? There is no consensus on timing or surgical strategy for the bidirectional superior cavopulmonary anastomosis.

The timing and exact surgical technique of a Fontan procedure has been the subject of much study. Some centers recommend performing the Fontan operation early; that is, at age 18 months to 2 years. Others recommend delaying application of a Fontan procedure for as long as possible to postpone the point at which the proverbial clock starts ticking with respect to the seemingly inevitable consequences of elevated central venous pressure. There is no consensus on the ideal age at which a patient should undergo a Fontan procedure. The fact that there are multiple techniques reported for performing a Fontan operation indicates that we have also not reached agreement on what the ideal Fontan connection should look like. The original atriopulmonary connection described by Fontan and $\mathrm{Kreutzer}^{1,2}$ has been essentially relegated to history. In its place there are currently primarily 2 types of procedures: lateral tunnel and extracardiac Fontan, both of which are forms of total cavopulmonary connection. In addition, there are variations on these, such as the intra/ extracardiac Fontan and the bifurcating graft Fontan. Then there is the question of whether or not the Fontan procedure should be fenestrated. Some centers recommend fenestrating all patients, some fenestrate no patients, and some leave a temporary fenestration. There is no consensus on the optimal surgical technique for the Fontan procedure.

Atrial arrhythmias following a Fontan procedure continue to occur despite the changes in surgical techniques. The treatment of atrial arrhythmias includes medical therapy, transcatheter ablation therapy, and the Fontan conversion procedure with arrhythmia surgery (ie, Cox maze III). The timing of when to recommend a patient with atrial arrhythmias for Fontan conversion is still unclear. What is clear is that many patients with an atriopulmonary Fontan connection who develop this condition have progressed to Fontan conversion, heart transplantation, or have not survived. The pool of candidates for this operation has rapidly diminished.

If a patient develops ventricular dysfunction, atrioventricular valve insufficiency, hepatic insufficiency, and/or protein-losing enteropathy he or she is now commonly referred for heart transplantation. The decision of when to list a patient for heart transplantation is still unclear. Mechanical assistance has been reported in only a handful of patients with varying results because of the complexity of the cardiac anatomy and the multiple operations before this intervention. There is no consensus on when to refer a failing Fontan patient for heart transplantation.

The Fontan operation has now been available to patients for more than 40 years. We have accomplished much in our quest for a better Fontan and have been able to apply this procedure to patients with increasingly complex presentations. Despite our best efforts, however, many patients still experience long-term sequelae, the prevention of which will only be possible by continued analysis of past experience. As noted earlier, this is a rapidly increasing population that will be a major user of resources at heart centers throughout the world.

Multi-institutional studies of as many patients as possible undergoing Fontan procedures may allow us to come to consensus on some of the as-yet-unresolved issues. This is a mission of the Society of Thoracic Surgeons Congenital Heart Surgery Database and the Congenital Heart Surgeons' Society Data Center. The articles featured in this issue of the Journal will certainly help to answer some of these questions. A midlife crisis may not be preventable, but it certainly can be modulated. But will we ever have consensus?

\section{References}

1. Fontan F, Baudet E. Surgical repair of tricuspid atresia. Thorax. 1971;26:240-8.

2. Kreutzer G, Galíndez E, Bono H, De Palma C, Laura JP. An operation for the correction of tricuspid atresia. J Thorac Cardiovasc Surg. 1973;66:613-21. 